\title{
A Ring Theoretic Construction of Hadamard Difference Sets in $\mathbb{Z}_{8}^{n} \times \mathbb{Z}_{2}^{n}$
}

\author{
XIANG-DONG HOU \\ xhou@ tarski.math.usf.edu \\ Department of Mathematics and Statistics, Wright State University, Dayton, Ohio 45435
}

Received August 22, 2002; Revised January 26, 2005; Accepted February 11, 2005

Abstract. Let $S=\operatorname{GR}\left(2^{3}, n\right)$ be the Galois ring of characteristic $2^{3}$ and rank $n$ and let $R=S[X] /\left(X^{2}, 2 X-4\right)$. We give an explicit construction of Hadamard difference sets in $(R,+) \cong \mathbb{Z}_{8}^{n} \times \mathbb{Z}_{2}^{n}$.

Keywords: bent function, finite Frobenius local ring, Galois ring, hadamard difference set

\section{Introduction}

Let $G$ be a finite group of order $v$. A subset $D \subset G$ is called a difference set in $G$ with parameters $(v, k, \lambda)$ if $|D|=k$ and $d_{1} d_{2}^{-1}\left(d_{1}, d_{2} \in D, d_{1} \neq d_{2}\right)$ represents each element in $G \backslash\{e\}$ exactly $\lambda$ times. A difference set with parameters $(v, k, \lambda)=\left(4 N^{2}, 2 N^{2}-\right.$ $N, N^{2}-N$ ) is called a Hadamard difference set. Initially studied by Menon [8], Hadamard difference sets have received much attention ever since. A lot is known about Hadamard difference sets: For example, in finite 2-groups, every nontrivial difference set is either a Hadamard difference set or a complement of a Hadamard difference set [8]. A finite abelian 2-group $G$ of order $2^{2 d+2}$ has a Hadamard difference if and only if $\exp (G) \leq d+2[10$, 6]. For a survey on Hadamard difference sets, the reader is referred to [2] by Davis and Jedwab.

The existence of Hadamard difference sets in abelian 2-groups with $|G|=2^{2 d+2}$ and $\exp (G) \leq d+2$ was proved by Kraemer [6]. The construction in [6] is algorithmic. There are still interests in more explicit constructions of Hadamard difference sets in abelian 2groups, as stated in one of the open problems in [2]. It seems that suitable ring structures on the groups are the key to explicit constructions. (The reader may see [3] and [4] for ring theoretic constructions of other types of difference sets.) In this note, we consider a finite ring $R=S[X] /\left(X^{2}, 2 X-4\right)$ where $S=\operatorname{GR}\left(2^{3}, n\right)$ is the Galois ring of characteristic $2^{3}$ and rank $n$ [7]. We give a simple and explicit construction of Hadamard difference sets in $(R,+) \cong \mathbb{Z}_{8}^{n} \times \mathbb{Z}_{2}^{n}$. 


\section{The construction}

Let $S=\operatorname{GR}\left(2^{3}, n\right)$ and

$$
R=S[X] /\left(X^{2}, 2 X-4\right)
$$

Denote the image of $X$ in $R$ by $x . R$ is a local ring with maximal ideal $2 R+x R$. Note that $2 R+x R$ is not a principal ideal, hence $R$ is not a chain ring [7]. However, $R$ has a unique minimal ideal $4 R$, hence $R$ is a finite Frobenius local ring [4]. In fact, the complete ideal lattice of $R$ is as follows:

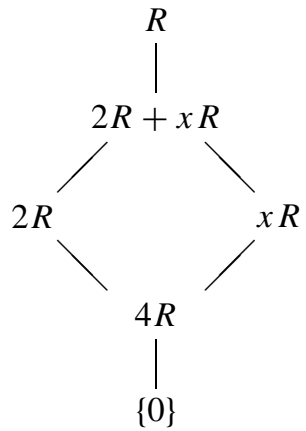

It is easy to see that $(R,+) \cong \mathbb{Z}_{8}^{n} \times \mathbb{Z}_{2}^{n}$ and that as an abelian group,

$$
(2 R+x R) / 4 R \cong \mathbb{Z}_{2}^{2 n}
$$

Let $\operatorname{Tr}: S \rightarrow \mathbb{Z}_{8}$ be the trace map of $S$. Define

$$
\begin{array}{lccc}
\lambda: & S[X] & \rightarrow & \mathbb{Z}_{8} \\
& a_{0}+a_{1} X+\cdots & \mapsto & \operatorname{Tr}\left(a_{0}+2 a_{1}\right)
\end{array}
$$

Then $\left(X^{2}, 2 X-4\right) \subset$ ker $\lambda$, hence $\lambda$ induces a $\mathbb{Z}_{8}$-linear map $\bar{\lambda}: R \rightarrow \mathbb{Z}_{8}$. Let $\xi=e^{2 \pi i / 8}$. Then $\chi()=\xi^{\bar{\lambda}()}$ is a character of $(R,+)$. Note that the minimal ideal $4 R \not \subset$ ker $\chi$. Hence $\chi$ is a generating character of $(R,+)$, i.e., every character of $(R,+)$ is of the form $\chi_{a}()=\chi(a \cdot)$ for some $a \in R[4]$. Let

$$
V=\{v \in 4 S: \operatorname{Tr}(v)=0\} .
$$

$V$ is an $(n-1)$-dimensional vector space over $\mathbb{Z}_{2}$. Note that

$$
\begin{aligned}
(S / 2 S) \times 4 S & \rightarrow 4 \mathbb{Z}_{8} \cong \mathbb{Z}_{2} \\
(a+2 S, v) & \mapsto \operatorname{Tr}(a v), \quad a \in S
\end{aligned}
$$


is a nondegenerate $\mathbb{Z}_{2}$-bilinear form. Thus

$$
\{a+2 S \in S / 2 S: \operatorname{Tr}(a v)=0 \text { for all } v \in V\}
$$

is a 1 -dimensional $\mathbb{Z}_{2}$-subspace of $S / 2 S$. Therefore, for $a \in S$,

$$
\operatorname{Tr}(a v)=0 \text { for all } v \in V \quad \text { iff } a \equiv 0 \text { or } 1 \quad(\bmod 2 S) .
$$

Let $T$ be the Teichmüller set of $S$ and put $T^{*}=T \backslash\{0\}$. Define

$$
D=T^{*}(1+x T+2 T+V) \subset R \backslash(2 R+x R) .
$$

Clearly, $|D|=\left(2^{n}-1\right) 2^{3 n-1}$. For any subgroup $H \subset(R,+)$, we use $H^{\perp}$ to denote the group of characters of $(R,+)$ which are principal on $H$. The following lemma gives the interesting character value distribution of $D$.

Lemma 2.1 Let $\psi$ be a nonprincipal character of $(R,+)$. We have

$$
\begin{cases}|\psi(D)|=2^{2 n-1}, & \text { if } \psi \notin(4 R)^{\perp}, \\ \psi(D)=0, & \text { if } \psi \in(4 R)^{\perp} \backslash(2 R+x R)^{\perp}, \\ \psi(D)=-2^{3 n-1}, & \text { if } \psi \in(2 R+x R)^{\perp} \backslash R^{\perp} .\end{cases}
$$

\section{Proof:}

Case 1. $\psi \notin(4 R)^{\perp}$. In this case, $\psi=\chi_{a}$ for some $a \in R^{\times}$, where $R^{\times}$is the multiplicative group of $R$ and $R^{\times}=T^{*}(1+x T+2 T+4 T)$. We may assume that $a=1+x b+2 c+4 d$ (b, $c, d \in T)$. Thus

$$
\begin{aligned}
\chi_{a}(D) & =\sum_{\substack{\epsilon \in T^{*}, v \in V \\
w, z \in T}} \chi(\epsilon(1+x w+2 z+v)(1+x b+2 c+4 d)) \\
& =\sum_{\substack{\epsilon \in T^{*} \\
w, z \in T}} \chi(\epsilon(1+x w+2 z)(1+x b+2 c+4 d)) \sum_{v \in V} \chi(\epsilon v) .
\end{aligned}
$$

It follows from (2.3) that

$$
\sum_{v \in V} \chi(\epsilon v)= \begin{cases}|V|, & \text { if } \epsilon=1, \\ 0, & \text { otherwise. }\end{cases}
$$


Hence

$$
\begin{aligned}
& \chi_{a}(D)=|V| \sum_{w, z \in T} \chi((1+x w+2 z)(1+x b+2 c+4 d)) \\
& \quad=|V| \sum_{w, z \in T} \chi(1+x b+2 c+4 d+x w+2 x w c+2 z+2 x z b+4 z c) \\
& \quad=|V| \sum_{w, z \in T} \chi(1+2 b+2 c+4 d+2 w+4 w c+2 z+4 z b+4 z c) \quad(\text { by }(2.1)) .
\end{aligned}
$$

Therefore,

$$
\left|\chi_{a}(D)\right|=|V|\left|\sum_{w \in T} \chi(2 w+4 w c)\right|\left|\sum_{z \in T} \chi(2 z+4(b+c) z)\right| .
$$

In the above,

$$
\begin{aligned}
\left|\sum_{w \in T} \chi(2 w+4 w c)\right| & =\left|\sum_{w \in T} \chi\left(2 w^{2}+4 w c\right)\right| \\
& =\left|\sum_{w \in T} \chi\left(2(w+c)^{2}\right)\right| \\
& =\left|\sum_{w \in T} \chi(2 w)\right| \\
& =2^{\frac{n}{2}},
\end{aligned}
$$

where the last step follows from the well known result about the exponential sum over the Teichmüller set of $\operatorname{GR}(4, n)[1,11]$. Of course, we also have $\left|\sum_{z \in T} \chi(2 z+4(b+c) z)\right|=$ $2^{n / 2}$. Therefore,

$$
\left|\chi_{a}(D)\right|=|V| 2^{n}=2^{2 n-1}
$$

Case 2. $\psi \in(4 R)^{\perp} \backslash(2 R+x R)^{\perp}$. In this case we may write $\psi=\chi_{a}$ where $a=x b+2 c+4 d$ (b, $c, d \in T, b$ and $c$ not both 0 ). We then have

$$
\begin{aligned}
\chi_{a}(D) & =\sum_{\substack{\epsilon \in T^{*}, v \in V \\
w, z \in T}} \chi(\epsilon(1+x w+2 z+v)(x b+2 c+4 d)) \\
& =|V| \sum_{\substack{\epsilon \in T^{*} \\
w, z \in T}} \chi(\epsilon(x b+2 c+4 d+2 x w c+2 x z b+4 z c)) \\
& =|V|\left[\sum_{\epsilon \in T^{*}} \chi(\epsilon(2 b+2 c+4 d))\right]\left[\sum_{w \in T} \chi(4 w c)\right]\left[\sum_{z \in T} \chi(4 z(b+c))\right] .
\end{aligned}
$$


At least one of $c$ and $b+c$ is nonzero. Thus

$$
\left[\sum_{w \in T} \chi(4 w c)\right]\left[\sum_{z \in T} \chi(4 z(b+c))\right]=0 .
$$

Case 3. $\psi \in(2 R+x R)^{\perp} \backslash R^{\perp}$. We can assume that $\psi=\chi_{4}$. Clearly,

$$
\chi_{4}(D)=|T|^{2}|V| \sum_{\epsilon \in T^{*}} \chi(4 \epsilon)=-|T|^{2}|V|=-2^{3 n-1} .
$$

Theorem 2.2 Let $E \subset(2 R+x R) / 4 R \cong \mathbb{Z}_{2}^{2 n}$ be any Hadamard difference set. Let $\bar{E} \subset$ $2 R+x R$ be the preimage of $E$. Then $D \cup \bar{E}$ is a Hadamard difference set in $(R,+)$.

Proof: First we have

$$
|D \cup \bar{E}|=|D|+|4 R||E|=\left(2^{n}-1\right) 2^{3 n-1}+2^{n}\left(2^{2 n-1}-2^{n-1}\right)=2^{4 n-1}-2^{2 n-1} .
$$

Let $\psi$ be any nonprincipal character of $(R,+)$. By the well known characterization of difference sets in abelian groups in terms of character values [10], we only have to show that $|\psi(D \cup \bar{E})|=2^{2 n-1}$. We have

$$
\psi(\bar{E})= \begin{cases}0, & \text { if } \psi \notin(4 R)^{\perp}, \\ \pm 2^{n} 2^{n-1}, & \text { if } \psi \in(4 R)^{\perp} \backslash(2 R+x R)^{\perp} \\ 2^{n}\left(2^{2 n-1}-2^{n-1}\right), & \text { if } \psi \in(2 R+x R)^{\perp} \backslash R^{\perp}\end{cases}
$$

Combining (2.4) and (2.5), we always have $|\psi(D \cup \bar{E})|=2^{2 n-1}$.

In the above construction, there are two independent pieces: a shell $D$ in $R \backslash(2 R+x R)$ and a core $\bar{E}$ in $2 R+x R$. We mention that this kind of shell-nesting method is common in constructions of Latin square type partial difference sets [5].

We compare the above construction with known constructions of Hadamard difference sets in finite abelian 2-groups. First, if the group is of the form $H \times H$, there is a very general construction of Hadamard difference sets using finite local rings [4]. However, when $n$ is odd, $\mathbb{Z}_{8}^{n} \times \mathbb{Z}_{2}^{n}$ is not of the form $H \times H$. Next, we consider the Menon construction [8]: Let $G_{1}$ and $G_{2}$ be finite groups and $D_{1} \subset G_{1}, D_{2} \subset G_{2}$. Then

$$
\left(D_{1} \times\left(G_{2} \backslash D_{2}\right)\right) \cup\left(\left(G_{1} \backslash D_{1}\right) \times D_{2}\right)
$$

is a Hadamard difference set in $G_{1} \times G_{2}$ if and only if $D_{i}$ is a Hadamard difference set in $G_{i}$ for $i=1,2$. When $G_{1} \neq 0$ and $G_{2} \neq 0$, we call a subset in $G_{1} \times G_{2}$ of the type (2.6) decomposable. 
Proposition 2.3 In Theorem 2.2, if $D \cup \bar{E}$ is decomposable in $(R,+)$, then $E$ is decomposable in $(2 R+x R) / 4 R \cong \mathbb{Z}_{2}^{2 n}$.

Proof: Assume that $R=G_{1} \times G_{2},\left(G_{i} \neq 0, i=1,2\right), D_{i} \subset G_{i}(i=1,2)$ and

$$
D \cup \bar{E}=\left(D_{1} \times\left(G_{2} \backslash D_{2}\right)\right) \cup\left(\left(G_{1} \backslash D_{1}\right) \times D_{2}\right) .
$$

Note that all elements in $D$ have order 8 and all elements in $\bar{E}$ have order $\leq 4$. Let $H_{i}=$ $\left\{g \in G_{i}: 4 g=0\right\}$ and put $F_{i}=D_{i} \cap H_{i}(i=1,2)$. Then $2 R+x R=H_{1} \times H_{2}$ and

$$
\bar{E}=\left(F_{1} \times\left(H_{2} \backslash F_{2}\right)\right) \cup\left(\left(H_{1} \backslash F_{1}\right) \times F_{2}\right) .
$$

We have

$$
\mathbb{Z}_{2}^{2 n} \cong \frac{2 R+x R}{4 R}=\frac{H_{1}}{4 G_{1}} \times \frac{H_{2}}{4 G_{2}}
$$

where $H_{i} / 4 G_{i} \neq 0(i=1,2)$. (Otherwise we would have $\operatorname{rank}\left(\frac{H_{1}}{4 G_{1}} \times \frac{H_{2}}{4 G_{2}}\right)<2 n$.) We claim that $F_{i}$ is a union of cosets of $4 G_{i}$ in $H_{i}(i=1,2)$. If $F_{i}=\varnothing$ or $H_{i}$ for some $i=1$ or 2 , the claim is obviously true. So assume that $F_{i} \neq H_{i}(i=1,2)$. Choose a nonprincipal character $\psi_{2}$ of $H_{2}$ such that $\psi_{2}\left(F_{2}\right) \neq 0$. Let $\psi_{1}$ be any character of $H_{1}$ which is not principal on $4 G_{1}$. Then $\psi_{1} \times \psi_{2}$ is a character of $H_{1} \times H_{2}=2 R+x R$ which is nonprincipal on $4 G_{1} \times 4 G_{2}=4 R$. Thus

$$
0=\left(\psi_{1} \times \psi_{2}\right)(\bar{E})=\psi_{1}\left(F_{1}\right) \psi_{2}\left(H_{2} \backslash F_{2}\right)+\psi_{1}\left(H_{1} \backslash F_{1}\right) \psi_{2}\left(F_{2}\right)=-2 \psi_{1}\left(F_{1}\right) \psi_{2}\left(F_{2}\right) .
$$

It follows that $\psi_{1}\left(F_{1}\right)=0$ for all $\psi_{1} \notin\left(4 G_{1}\right)^{\perp}$. Therefore $F_{1}$ is a union of cosets of $4 G_{1}$ in $H_{1}$. In the same way, $F_{2}$ is a union of cosets of $4 G_{2}$ in $H_{2}$. Mapping both sides of (2.7) to $\frac{2 R+x R}{4 R}=\frac{H_{1}}{4 G_{1}} \times \frac{H_{2}}{4 G_{2}}$, we have

$$
E=\left[\tilde{F}_{1} \times\left(\frac{H_{2}}{4 G_{2}} \backslash \tilde{F}_{2}\right)\right] \cup\left[\left(\frac{H_{1}}{4 G_{1}} \backslash \tilde{F}_{1}\right) \times \tilde{F}_{2}\right]
$$

where $\tilde{F}_{i}$ is the image of $F_{i}$ in $H_{i} / 4 G_{i}$. Thus $E$ is decomposable.

Hadamard difference sets in $\mathbb{Z}_{2}^{2 n}$ are precisely supports of bent functions on $\mathbb{Z}_{2}^{2 n}$ [9]. There are many indecomposable bent functions. For example, any bent function on $\mathbb{Z}_{2}^{2 n}$ of degree $n$ is indecomposable [9]. Choose any indecomposable bent function on $\mathbb{Z}_{2}^{2 n}$ and let $E$ be the corresponding indecomposable Hadamard difference set in $\mathbb{Z}_{2}^{2 n}$. Then by Proposition 2.3, the Hadamard difference set $D \cup \bar{E}$ in Theorem 2.2 is indecomposable hence can not be obtained from the Menon construction.

The construction in [6] works for all abelian groups $G$ with $|G|=2^{2 d+2}$ and $\exp (G) \leq$ $d+2$. However, we find it difficult to compare the constructions in this note and in [6] because of the algorithmic nature of the latter. 


\section{References}

1. S. Boztaş, R. Hammons, and P.V. Kumar “4-phase sequences with near-optimum correlation properties,” IEEE Trans. Inform. Theory 38 (1992), 1101-1113.

2. J.A. Davis and J. Jedwab, "A survey of Hadamard difference sets," in Groups, Difference Sets, and the Monster, K. T. Arasu et al. (Eds.), de Gruyter, New York, 1996, pp. 145-156.

3. X. Hou, "Bent functions, partial difference sets and quasi-Frobenius local rings," Des. Codes Cryptogr. 20 (2000), 251-268.

4. X. Hou, "Rings and constructions of partial difference sets," Discrete Math. 270 (2003), 149-176.

5. X. Hou and A. Nechaev, A construction of finite Frobenius rings and its applications to partial difference sets, preprint.

6. R.G. Kraemer, "Proof of a conjecture on Hadamard 2-groups," J. Combin. Theory A 63 (1993), 1-10.

7. B.R. McDonald, Finite Rings with Identity, Marcel Dekker, New York, 1974.

8. P.K. Menon, "On difference sets whose parameters satisfy a certain relation," Proc. Amer. Math. Soc. 13 (1962), 739-745.

9. O.S. Rothaus, "On "bent" functions," J. Combin. Theory A 20 (1976), 300-305.

10. R.J. Turyn, "Character sums and difference sets," Pacific J. Math. 15 (1965), 319-346.

11. K. Yang, T. Helleseth, and P.V. Kumar, "On the weight hierarchy of Kerdock codes over $\mathbb{Z}_{4}$," IEEE Trans. Inform. Theory 42 (1996), 1587-1593. 